\section{"Medical audit of rectal biopsy diagnosis of inflammatory bowel disease" is not medical audit}

Dr Morson kindly sent me a prepublication copy of this article. ${ }^{1}$ It is an important paper, in which the authors studied the prognostic reliability of biopsies and the biological behaviour of colorectal disease. This is not remotely an approach to medical audit as suggested in the last paragraph.

In chemical pathology we already go three stages further towards medical audit : (a) in (internal) quality control a laboratory repeatedly analyses the same specimen to see if it gets the same answer; (b) in (external) quality assurance the same specimen is analysed by many different laboratories, and comparison is made of their results; (c) an advisory panel is available to give confidential help to laboratories that are persistently poor performers. There are similar procedures in haematology and in medical microbiology. ${ }^{2}$

Histopathology has barely started on such assessments, and I think the authors missed an opportunity. Why did they not (i) have their staff look at the same specimens several times, without knowing that these were the same specimens? (ii) send the specimens to a number of unselected different histopathologists, and compare their reports? These two procedures could have been a beginning of medical audit applied to histopathology.

DN BARON

Department of Chemical Pathology, Royal Free Hospital and School of Medicine, London NW3 2QG

\section{References}

${ }^{1}$ Frei JV, Morson BC. Medical audit of rectal biopsy diagnosis of inflammatory bowel disease. J Clin Pathol 1982;35:3414.

${ }^{2}$ Baron DN. The improvement of performance in pathology. In: McLachlan $\mathrm{G}$, ed. Reviewing practice in medical care; steps to quality assurance. London: Nuffield Provincial Hospitals Trust, 1981:57-68.

Drs Morson and Frei reply as follows:

Professor Baron's comment fails to recognise the essential difference between histopathology and other branches of laboratory medicine particularly chemical pathology. Identical aliquots, objective instrumental evaluation, mean and standard deviations do not exist for pieces of tissue as they do for tubes of blood. We studied the consistency of written reports, not the specimen itself. In other words, we examined the implications of reporting rectal biopsies in inflammatory bowel disease as they were documented in the departmental records. It was a retrospective research into the accuracy of reporting in the hope that further study will lead to improved performance. Professor Baron proposes an altogether different study involving review of specimens rather than records.

But what constitutes medical audit? We are not qualified to disagree with Professor Baron's definition of how audit should be conducted for chemical pathology, but he must accept that it is at present defined differently to suit the varying circumstances of medical practice. The general objectives of medical audit are to investigate standards and improve the performance of individual practitioners. In the first place, it is essentially a comparison of what has happened with what ought to happen. But medical audit is not complete unless, as a second stage, it leads to an appropriate change for the better.

We maintain that our article is essentially a first stage audit and that hopefully further research will lead to higher accuracy rates for rectal biopsy diagnosis in non-specific inflammatory bowel disease. In histopathology audit can be prospective but more often retrospective, as in clinical medicine, and relies on a degree of uniformity in departmental record systems. It is little different in our opinion from what is generally included under the heading of "clinical research" except that medical audit should be a continuing and organised curiosity into how we are performing on both an intraand interdepartmental basis. A good example of the latter was reported recently ${ }^{1}$ and subsequently discussed in an editorial in the British Medical Journal. ${ }^{2}$

BC MORSON JV FREI

Department of Pathology, St Mark's Hospital, City Road, London ECVI 2PS
References

${ }^{1}$ Blenkinsopp WK, Stewart-Brown $S$, 음 Blesovsky L, Keemeg G, Fielding LP. Histopathology reporting in large bowel $\mathbb{D}$ cancer. J Clin Pathol 1981;34:509-13.

${ }^{2}$ Morson BC. Histopathology reporting in large-bowel cancer. Br Med J 1981;283: $\overrightarrow{0}$ 1493.

\section{Possible diurnal variation in} 2,3-diphosphoglyceric acid

The importance of 2,3-diphosphoglyceric is acid (2,3-DPG) as a regulator of overall is oxygen affinity of haemoglobin $(\mathrm{Hb})$ in $/ 2$ human erythrocytes is well established; $1^{1-3}$ ? it is an important mechanism by which the 을 body controls the oxygen supply to the $\rightarrow$ tissues.

The purpose of the present study was to investigate whether 2,3-DPG concentrations show any diurnal variation which would need to be considered in future work; no information on this point could be found.

\section{SUBJECTS AND METHODS}

Five apparently healthy, non-smoking male medical students volunteered to take part in this study. Blood samples were withdrawn by venepuncture into heparinised vacuum tubes at $08.00,12.00,17.00$ and 00.00 hours.

Samples were assayed for 2,3-DPG according to the ultraviolet enzymatic method of Sigma Chemical $\mathrm{Co} ;{ }^{4}$ the method uses 2-phosphoglycolic acid as a stimulator, and is similar to that of Lowry et al, ${ }^{5}$ but the reaction is monitored spectrophotometrically instead of fluorometrically: 3-phosphoglycerate phosphokinase (PGK) and glyceraldehyde-3phosphate dehydrogenase (GAPD) are coupled to convert 3-phosphoglycerate (3-PGA) to glyceraldehyde-3-phosphate (G-3-P), with simultaneous formation of NAD from NADH. The concentration of 2,3-DPG is proportional to the amount of NADH oxidised; calculations are based on the molar extinction coefficient of NADH.

In this procedure, the test is performed on a protein-free supernatant; this was prepared as quickly as possible, using cold $8 \%$ trichloroacetic acid (TCA), and stored frozen until analysed. Samples for each subject were assayed in a single batch; 
each sample was tested in duplicate.

A limited study of the within-batch precision of the assay was carried out. Using a sample containing 2,3-DPG at a level of $2 \cdot 36 \mu \mathrm{mol} / \mathrm{cm}^{3}$ for eight analyses, the range of values was 2.31 to $2 \cdot 39$ $\mu \mathrm{mol} / \mathrm{cm}^{3}$, with a standard deviation (SD) of $0.04 \mu \mathrm{mol} / \mathrm{cm}^{3}$ and a coefficient of variation (CV) of $2 \%$. These figures are in keeping with published data. ${ }^{4}$

Haemoglobin levels were measured by means of a Coulter Counter (model S).

\section{RESULTS AND DISCUSSION}

The results of the study are presented in the Table. It can be seen that in these subjects no significant diurnal variation was observed. We had anticipated that the haemodilution which occurs in subjects whilst in the supine position ${ }^{6}$ might affect 2,3-DPG concentrations if expressed in terms of $\mu \mathrm{mol} / \mathrm{cm}^{3}$ of whole blood; however, such haemodilution was not demonstrated in these subjects- $\mathrm{Hb}$ concentrations in the samples taken at 08.00 and 00.00 hours were very similar.

According to Hagan et $a l,{ }^{6}$ haemoconcentration is associated with movement to a standing position, and stability is not achieved for $20 \mathrm{~min}$. With this in mind, our volunteers presented themselves for venepuncture immediately on rising at 08.00 , but we still failed to demonstrate haemodilution in this group of subjects.

Concentrations of 2,3-DPG appeared to vary slightly with $\mathrm{Hb}$ concentration, but considering the precision of the assay method, the variations were so small that they were regarded as negligible. Hence we concluded that, in the small population studied no diurnal variation in 2,3-DPG was shown.

We should like to thank Professor JW Stewart and the staff of the Haematology Department for their co-operation with this study.

SHEILA AGATE AL MILLER Courtauld Institute of Biochemistry, Middlesex Hospital Medical School, London WIP TPN

\section{References}

' Chanutin A. Churnish RR. Effect of organic and inorganic phosphates on the oxygen equilibrium of human erythrocytes. Arch Biochem Biophy's 1967;121: 96-102.

2 Benesch R. Benesch RE. The effect of organic phosphates from the human erythrocyte on the allosteric properties of haemoglobin. Biochem Biophys Res Commun 1967;26:162-7.

${ }^{3}$ Eaton JW, Brewer GJ. The relationship between red cell 2,3-diphosphoglycerate and levels of haemoglobin in the human. Proc Natl Acad Sci USA 1968;61:75660.

' Sigma Technical Bulletin No 35-UV (Sigma Chemical Co, Fancy Road. Poole, Dorset $\mathrm{BH} 17$ 7NH).

Possible diurnal variation in 2,3-diphosphoglyceric acid

\begin{tabular}{lllll}
\hline Sample* & Time $(h)$ & $H b(\mathrm{~g} / \mathrm{dl})$ & $2,3-D P G\left(! \mathrm{mol} / \mathrm{cm}^{3}\right)$ & $2,3-D P G(! \mathrm{mol} / \mathrm{gHb})$ \\
\hline $\mathrm{A}_{1}$ & 08.00 & $15 \cdot 5$ & $2 \cdot 16$ & $13 \cdot 9$ \\
$\mathrm{~A}_{2}$ & 12.00 & $15 \cdot 2$ & $2 \cdot 00$ & $13 \cdot 2$ \\
$\mathrm{~A}_{3}$ & 17.00 & $15 \cdot 4$ & $2 \cdot 08$ & $13 \cdot 5$ \\
$\mathrm{~A}_{4}$ & 00.00 & $15 \cdot 3$ & $2 \cdot 08$ & $13 \cdot 6$ \\
$\mathrm{~B}_{1}$ & 08.00 & $15 \cdot 4$ & $2 \cdot 31$ & $15 \cdot 0$ \\
$\mathrm{~B}_{2}$ & 12.00 & $15 \cdot 3$ & $2 \cdot 23$ & $14 \cdot 6$ \\
$\mathrm{~B}_{3}$ & 17.00 & $15 \cdot 1$ & $2 \cdot 16$ & $14 \cdot 3$ \\
$\mathrm{~B}_{4}$ & 00.00 & $15 \cdot 2$ & $2 \cdot 16$ & $14 \cdot 2$ \\
& & & & $13 \cdot 8$ \\
$\mathrm{C}_{1}$ & 08.00 & $15 \cdot 7$ & $2 \cdot 16$ & $14 \cdot 1$ \\
$\mathrm{C}_{2}$ & 12.00 & $15 \cdot 8$ & $2 \cdot 23$ & $13 \cdot 9$ \\
$\mathrm{C}_{3}$ & 17.00 & $15 \cdot 5$ & $2 \cdot 16$ & $14 \cdot 3$ \\
$\mathrm{C}_{4}$ & 00.00 & $16 \cdot 1$ & $2 \cdot 31$ & $14 \cdot 6$ \\
$\mathrm{D}_{1}$ & 08.00 & $15 \cdot 8$ & $2 \cdot 31$ & $13 \cdot 3$ \\
$\mathrm{D}_{2}$ & 12.00 & $16 \cdot 2$ & $2 \cdot 16$ & $14 \cdot 5$ \\
$\mathrm{D}_{3}$ & 17.00 & $15 \cdot 9$ & $2 \cdot 31$ & $15 \cdot 0$ \\
$\mathrm{D}_{4}$ & 00.00 & $15 \cdot 9$ & $2 \cdot 39$ & $13 \cdot 8$ \\
$\mathrm{E}_{1}$ & 08.00 & $16 \cdot 8$ & $2 \cdot 31$ & $12 \cdot 7$ \\
$\mathrm{E}_{2}$ & 12.00 & $17 \cdot 6$ & $2 \cdot 23$ & $13 \cdot 0$ \\
$\mathrm{E}_{3}$ & 17.00 & $17 \cdot 1$ & $2 \cdot 23$ & $13 \cdot 8$ \\
$\mathrm{E}_{1}$ & 00.00 & $16 \cdot 9$ & $2 \cdot 31$ & \\
\hline & & & & \\
\hline
\end{tabular}

*Subjects were allocated the letters A, B, C, D and E, s Lowry OH. Passoneau JV, Hasselberge FX. Schulz DW. Effect on ischemia on: known substrates and cofactors of the glycolytic pathway in brain. J Biof Chem 1964;239:18-30.

${ }^{6}$ Hagan RD, Diaz FJ. Horvath SM. Plasm: volume changes with movement $t \overline{\bar{e}}$ supine and standing positions. $J A p p \vec{p}$ Phisiol 1978:45, 3:414-8.

Errors in weighing when using a single-par balance

The errors in weighing plastic vials on के single-pan balance have been noted ${ }^{1}$ andP the cause attributed to changes in the् electrostatic charges on the vials whenis handling them with plastic disposables gloves. In the same study, smaller errors of̂ up to $0.44 \mathrm{mg}$ were found when weighing glass vials and these were attributed to general errors in weighing. The lattererrors appear to be excessive.

Weighing a $10 \mathrm{~g}$ weight on a single-par five-place balance (the Mettler H54AR) the maximum difference I found between $\infty$ any two weighings within batch was 0.01 N $^{-}$ mg (Table 1).

Using the same balance to weigh glass vials I obtained comparable precision? values when plastic forceps were used tom handle the vials (Table 2). Precision deteriorated however when the glass vials were handled otherwise; the worst result $\overrightarrow{\bar{\delta}}$ were obtained when the glass vials were handled with plastic gloves especially? when more than minimal handling waso involved (maximum weight change 0.78 $\mathrm{mg}$ ). Plastic vials could be weighed precisely when plastic forceps were used too handle them although the error involved. was larger than that found for glass viali (Table 2). Handling plastic vials with plastic gloves resulted in much lar£ero errors (maximum weight change $+2 \cdot 47$ $\mathrm{mg}$ ).

Whilst the above results are in agreemene with those found by Fleck et al, it wor:lo appear that the errors they noted wher weighing glass vials were not due too general errors in weighing but were caused by using plastic gloves to handle the vialso which presumably alters some physicat characteristic of the vials such as the electrostatic charges on them. In supports of this, my own experiments were designed to minimise any effect due to fluctuations in external factors such as temperature, humidity and atmospheri@ pressure. Furthermore different technique: of handling produced different sizes of changes in apparent weight and these 